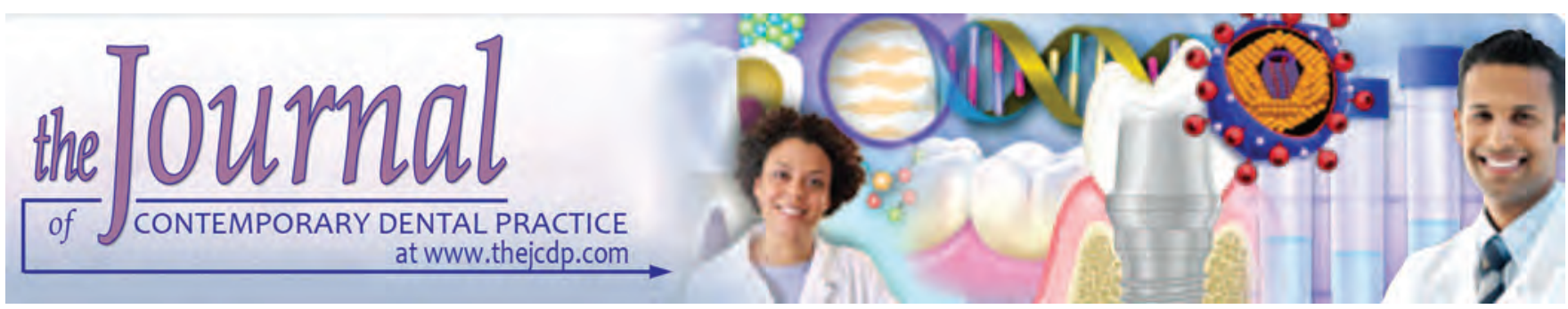

\title{
A Clinicopathologic Study of Oral Changes in Gastroesophageal Reflux Disease, Gastritis, and Ulcerative Colitis
}

${ }^{1} \mathrm{E}$ Vinesh, ${ }^{2} \mathrm{KMK}$ Masthan, ${ }^{3} \mathrm{M}$ Sathish Kumar, ${ }^{4} \mathrm{~S}$ Marytresa Jeyapriya, ${ }^{5}$ Aravindha Babu, ${ }^{6}$ Meera Thinakaran

\begin{abstract}
Objectives: The aim and objectives of this study are to identify oral changes in certain gastrointestinal (GI) diseases, namely gastroesophageal reflux disease (GERD), ulcerative colitis, gastritis, and to evaluate these oral symptoms as indicators for assessing $\mathrm{Gl}$ disorders.
\end{abstract}

Materials and methods: In this study, the oral manifestations of various $\mathrm{GI}$ disorders were assessed in a varying age group of 250 patients in Government Stanley Medical College and Hospital, Chennai. Out of 250 patients, 142 were affected by GERD, 99 were affected by gastritis, and 9 patients were affected by ulcerative colitis. Of these patients, 177 were males and 73 were females.

Results: Evaluation of patients with gastritis revealed that $66.7 \%$ affected with gingivitis, $19.2 \%$ with dental erosion on the palatal and lingual aspects of maxillary and mandibular teeth predominantly in the anterior region, $10.1 \%$ with periodontitis, $2 \%$ with gingival erythema. Among the patients with GERD, $44 \%$ of the cases showed dental erosion, $25.5 \%$ periodontitis, $9.9 \%$ gingivitis, 5.7\% gingival erythema, 2.8\% palatal erythema, 2.1\% gingival ulcers, glossitis $2 \%, 1.4 \%$ floor of the mouth erythema, and $0.7 \%$ erythema of the tongue. Patients with ulcerative colitis showed $44.4 \%$ of gingival erythema, $33.3 \%$ of dental erosions, and $22.2 \%$ of gingival ulcers and periodontitis.

Conclusion: In our study of 250 patients, oral manifestations were observed in $88 \%$ of the patients. Both soft tissue and hard tissue changes were evident. There was a high correlation

1,3,4 Department of Oral Pathology, Karpaga Vinayaga Institute of Dental Sciences, Kanchipuram, Tamil Nadu, India

2,5 Department of Oral Pathology, Sree Balaji Dental College and Hospital, Chennai, Tamil Nadu, India

${ }^{6}$ Department of Oral and Maxillofacial Surgery, Karpaga Vinayaga Institute of Dental Sciences, Kanchipuram, Tamil Nadu, India

Corresponding Author: Vinesh E, Department of Oral Pathology Karpaga Vinayaga Institute of Dental Sciences, Kanchipuram Tamil Nadu, India, e-mail: vineshezhil@gmail.com between various GI disease and dental erosion, erythema at various sites of the oral cavity, oral ulcers, gingivitis, periodontitis, and glossitis. Careful evaluation of oral cavity may unveil many $\mathrm{Gl}$ disorders and help the patient by providing early diagnosis, which further facilitates the prognosis.

Keywords: Gastritis, Gastroesophageal reflux disease, Ora manifestations of gastrointestinal disorders, Ulcerative colitis.

How to cite this article: Vinesh E, Masthan KMK, Kumar MS, Jeyapriya SM, Babu A, Thinakaran M. A Clinicopathologic Study of Oral Changes in Gastroesophageal Reflux Disease, Gastritis, and Ulcerative Colitis. J Contemp Dent Pract 2016;17(11):943-947.

Source of support: Nil

Conflict of interest: None

\section{INTRODUCTION}

The oral cavity, lined by stratified squamous epithelium, is the portal of entry to the gastrointestinal (GI) tract, and as a consequence, it is often involved in conditions that affect the GI tract. ${ }^{1}$

Gastrointestinal diseases manifest as lesions within the jaws, oral mucosa, or perioral tissues. These lesions are directly caused by GI diseases or at times, by systemic alterations secondary to GI diseases. ${ }^{2}$

Gastroesophageal reflux disease (GERD) is defined as a "condition that develops when the reflux of stomach contents causes troublesome symptoms and/or complications." ${ }^{3}$ The common symptoms of GERD include heartburn, regurgitation, and dysphagia. ${ }^{4}$ Oral manifestations of GERD include dental erosion, dental caries, xerostomia, burning sensation, halitosis, and erythema of the palatal mucosa and uvula. ${ }^{5}$

Ulcerative colitis is a type of inflammatory bowel disease that primarily affects the colonic mucosa. Initial symptoms include diarrhea with blood and mucus, fever, and weight loss. ${ }^{6}$ Oral manifestations include aphthous ulceration and pyostomatitis vegetans. ${ }^{7}$ 


\begin{tabular}{lll}
\hline \multicolumn{3}{c}{ Table 1: Oral manifestations in patients with GERD } \\
\hline Oral manifestations & No. of patients & \% of \\
Tooth erosion & (Frequency) & occurrence \\
Periodontitis & 63 & 44.6 \\
Gingivitis & 36 & 25.5 \\
Gingival erythema & 14 & 9.9 \\
Palatal erythema & 8 & 5.7 \\
Gingival ulcers & 4 & 2.8 \\
Glossitis & 3 & 2.1 \\
Erythema in floor of the mouth & 2 & 2.1 \\
Tongue erythema & 1 & 1.4 \\
\hline
\end{tabular}

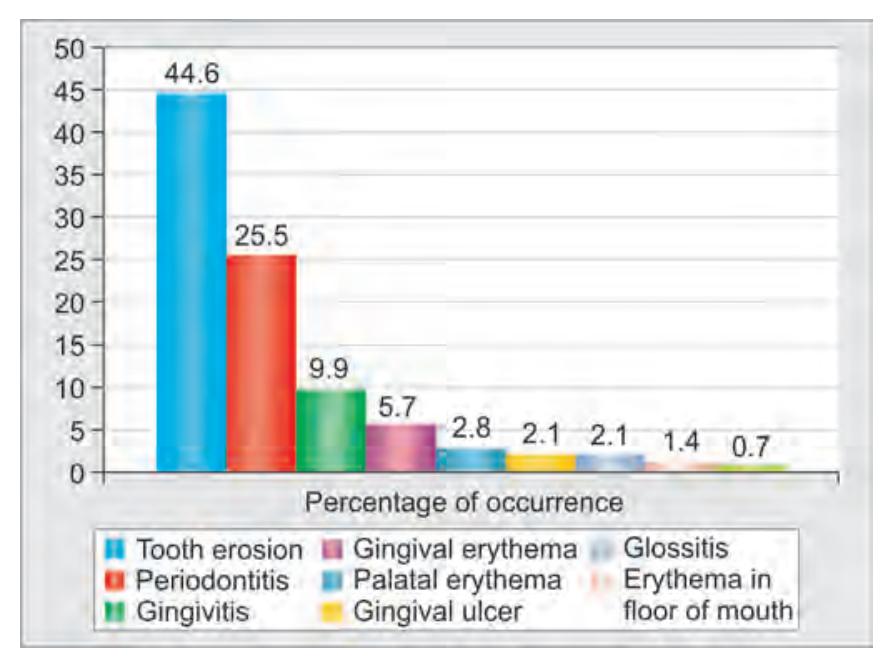

Graph 1: Occurrence of oral manifestations in GERD

The purpose of this study is to show that oral cavity is a mirror that reflects the underlying GI diseases and also to prove that mouth could be the mirror of various GI diseases. The aim and objectives of this study are to identify oral changes in certain GI diseases, namely GERD, ulcerative colitis, gastritis, and to evaluate these oral symptoms as indicators for assessing GI disorders.

\section{MATERIALS AND METHODS}

In this study, the oral manifestations of various GI disorders were assessed in a varying age group of 250 patients in Government Stanley Medical College and Hospital, Chennai.

Out of 250 patients, 142 were affected by GERD, 99 were affected by gastritis, and 9 patients were affected by ulcerative colitis. Of these patients, 177 were males and 73 were females.

\section{RESULTS}

As shown in Table 1, the classical intraoral finding in 142 patients ( 99 males and 43 females) affected by GERD was dental erosion in 63 patients $(44.6 \%)$. The next common finding was periodontitis in 36 patients (25.5\%). This was
Table 2: Oral manifestations in patients with gastritis

\begin{tabular}{lll}
\hline & $\begin{array}{l}\text { No. of patients } \\
\text { (Frequency) }\end{array}$ & \% of occurrence \\
\hline Gingivitis & 66 & 66.7 \\
Tooth erosion & 19 & 19.2 \\
Periodontitis & 10 & 10.1 \\
Gingival erythema & 2 & 2 \\
\hline
\end{tabular}

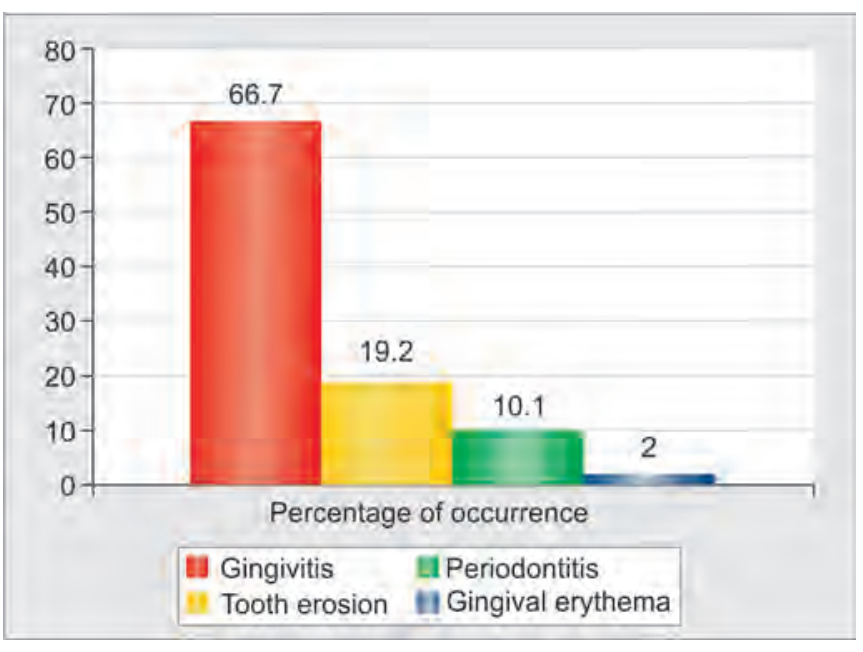

Graph 2: Occurrence of oral manifestations in gastritis

followed by gingivitis in 14 patients (9.9\%). Eight (5.7\%), $4(2.8 \%), 3(2.1 \%), 3(2.1 \%), 2(1.4 \%)$, and $1(0.7 \%)$ were the numbers of patients affected by gingival erythema, palatal erythema, gingival ulcer, glossitis, floor of the mouth erythema, and tongue erythema respectively. Eight patients did not show any oral manifestations (Graph 1).

As shown in Table 2, the classical intraoral finding in 99 patients (70 males and 29 females) affected by gastritis was gingivitis in 66 patients $(66.7 \%)$ followed by dental erosion in 19 patients (19.2\%), and periodontitis in 10 patients (10.1\%) (Graph 2).

As shown in Table 3, the classical intraoral finding in 9 patients ( 8 males and 1 female) affected by ulcerative colitis was gingival erythema in 4 patients $(44.4 \%)$, this was followed by dental erosion in 3 patients (33.3\%). Gingival ulcer and periodontitis were seen in 2 patients each (22.2\%) (Graph 3).

\section{DISCUSSION}

The oral cavity is an important anatomical location where many critical physiologic processes, such as digestion, respiration, and speech, take place. The oral cavity is frequently involved by conditions that affect multiple organs of the body. Anatomically and functionally, the oral cavity represents the beginning of the GI tract. The disease processes affecting the GI tract may cause observable changes in the oral cavity and sometimes, oral involvement precedes the GI symptoms. ${ }^{1}$

In our study of 250 patients, various GI conditions like gastritis, GERD, and ulcerative colitis were evaluated for 


\begin{tabular}{lll}
\multicolumn{2}{c}{ Table 3: Oral manifestations in patients with ulcerative colitis } \\
\hline & No. of patients & \% of \\
Oral manifestations & (Frequency) & occurrence \\
\hline Gingival erythema & 4 & 44.4 \\
Tooth erosion & 3 & 33.3 \\
Periodontitis & 2 & 22.2 \\
Gingival ulcers & 2 & 22.2 \\
\hline
\end{tabular}

its oral manifestations. Among the study population, $88 \%$ of the patients showed oral symptoms, such as dental erosions, erythema, periodontitis, gingivitis, gingival ulcers, and glossitis.

Evaluation of patients with gastritis revealed $66.7 \%$ affected with gingivitis, $19.2 \%$ with dental erosion on the palatal and lingual aspects of maxillary and mandibular teeth predominantly in the anterior region, $10.1 \%$ with periodontitis, $2 \%$ with gingival erythema. Dye et al ${ }^{8}$ and $\mathrm{Al}$ Asqah et $\mathrm{al}^{9}$ in their study showed Helicobacter pylori bacteria present in periodontal lesions of the gastritis patients, which play a major role in the pathogenesis of the disease. Their study highlights the association of periodontitis with

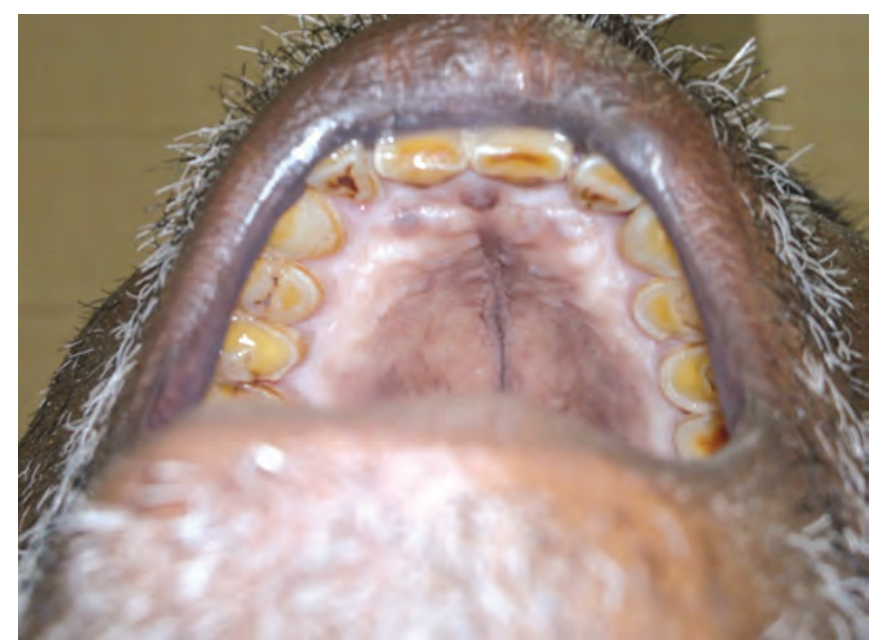

Fig. 1: Maxillary dental erosions

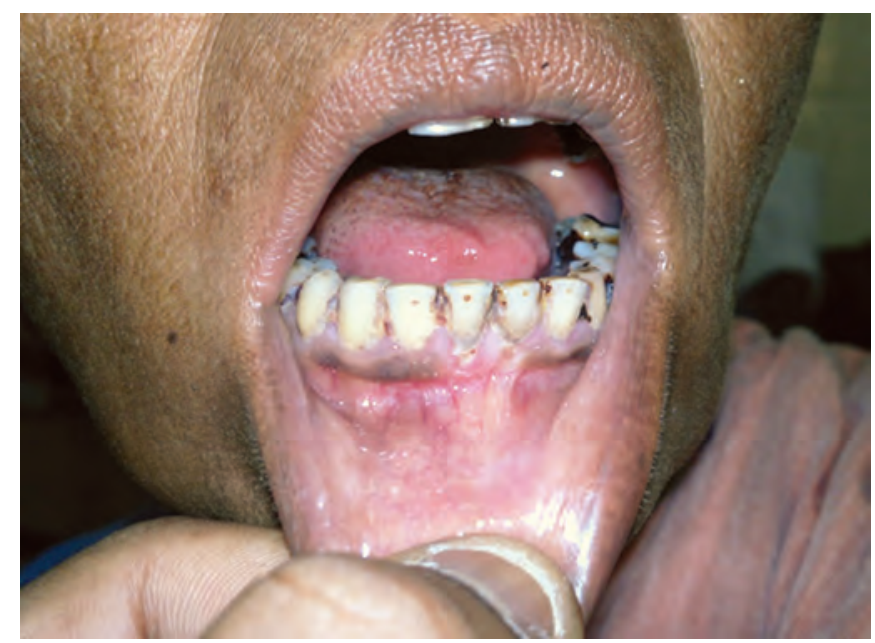

Fig. 3: Gingivitis

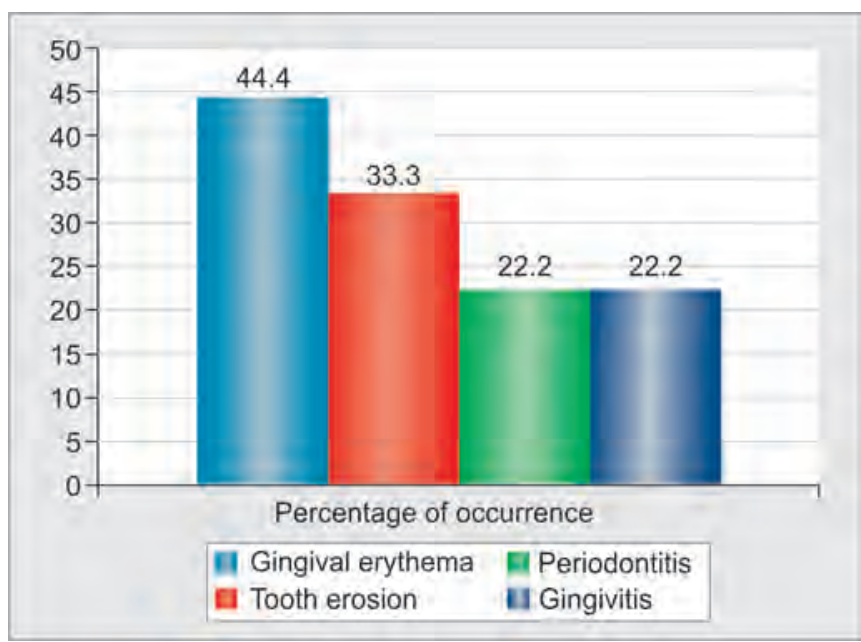

Graph 3: Occurrence of oral manifestations in ulcerative colitis

gastritis which correlates with our study. However, there are not many studies in the literature regarding the other oral manifestations of gastritis, like dental erosion, which our study attempts to establish it (Figs 1 to 6).

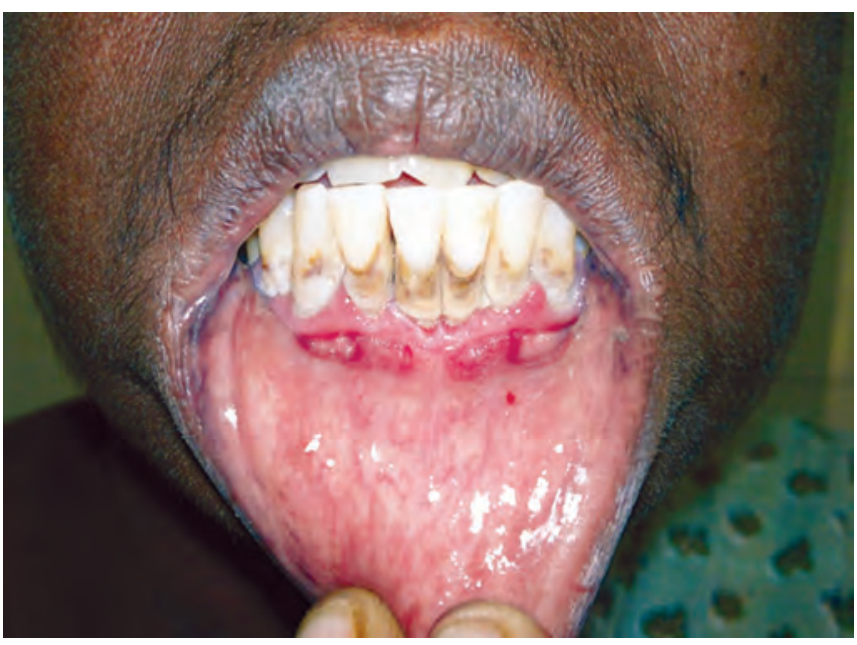

Fig. 2: Gingival ulcers and periodontitis

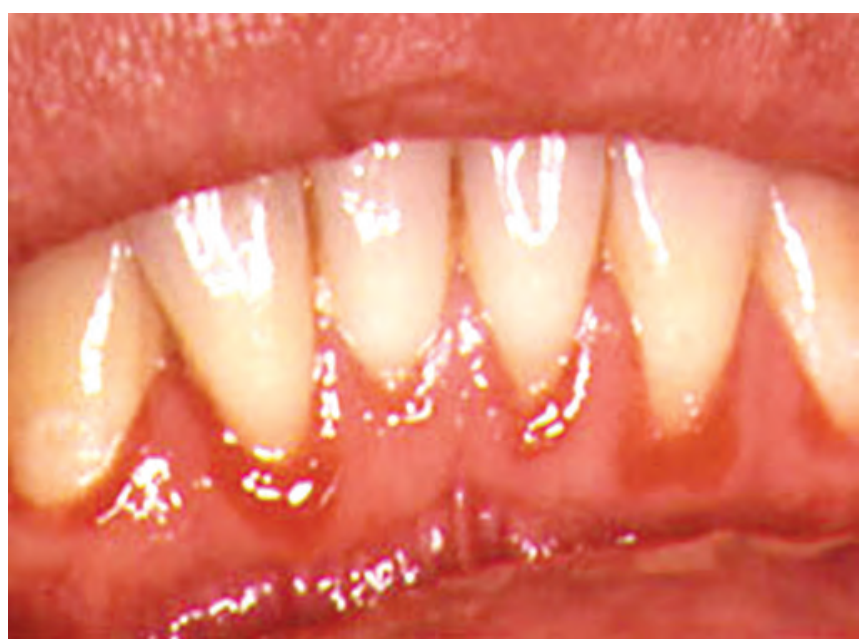

Fig. 4: Gingival erythema 


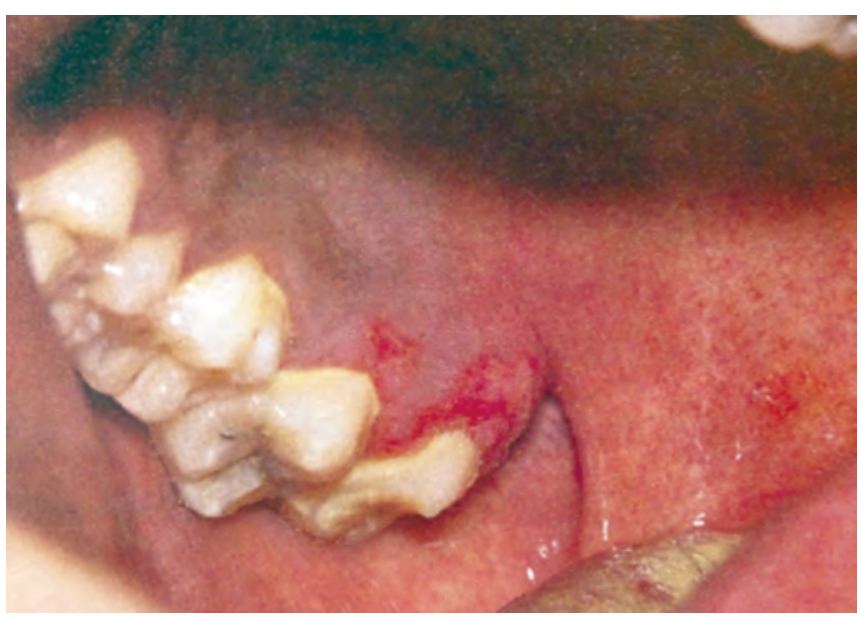

Fig. 5: Palatal erythema

Among the patients with GERD, $44 \%$ of the cases showed dental erosion, $25.5 \%$ periodontitis, $9.9 \%$ gingivitis, $5.7 \%$ gingival erythema, $2.8 \%$ palatal erythema, $2.1 \%$ gingival ulcers, glossitis $2 \%, 1.4 \%$ floor of the mouth erythema, and $0.7 \%$ erythema of the tongue. The relationship between GERD and dental erosion correlated with the study by Schroeder et al ${ }^{10}$ and Barron et al. ${ }^{11}$

Fan-Hsu in his study had mentioned that crucial examination of the patient with dental erosion may reveal silent GERD, thereby he strongly supports the association between dental erosion and GERD. ${ }^{12}$ Daley et $\mathrm{al}^{2}$ in their review suggested that enamel erosion is caused by gastric acids in gastric reflux patients and the erosion is mostly seen on the broad areas of the teeth. Although Huber in his review had recorded the occurrence of oral ulcers and glossitis, ${ }^{13}$ the significance of other oral findings in our study was not supported by any literature.

Patients with ulcerative colitis showed $44.4 \%$ of gingival erythema, $33.3 \%$ of dental erosions, $22.2 \%$ of gingival ulcers and periodontitis. Similar findings have been reported by Schroeder who demonstrated 10 out of 12 ulcerative colitis patients with significant dental erosion. ${ }^{14}$ Trost and McDonnell ${ }^{15}$ highlighted that oral ulcers in colitis disease were seen especially localized to gingiva and floor of the mouth. Galbraith et $\mathrm{al}^{16}$ proved that oral ulcerations were definitive manifestations of ulcerative colitis and these ulcers were mostly found in the gingiva. Van Dyke et $\mathrm{al}^{17}$ isolated microorganisms, such as Wolinella and Campylobacter from patients with inflammatory bowel disease and from the associated periodontal lesions, thereby they proved that these microorganisms, colonizing in the periodontal lesions of the ulcerative colitis patients, play a major role in the pathogenesis of the disease. Thus they justify the relationship between periodontitis and ulcerative colitis. Katz et $\mathrm{al}^{18}$ in their study of oral signs and symptoms of ulcerative colitis, patients with active disease showed oral ulcerations. Jose et $\mathrm{al}^{19}$ in his article suggested that oral ulcers were seen in 5 to $10 \%$ of patients

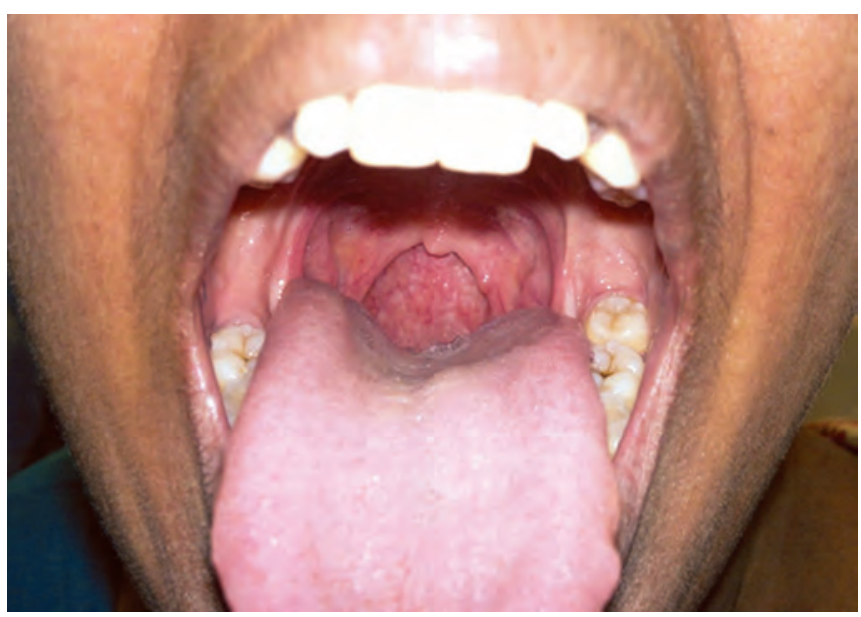

Fig. 6: Erythema of soft palate

affected by ulcerative colitis. Franch et $\mathrm{al}^{20}$ suggested that oral manifestations of ulcerative colitis may manifest as aphthous ulcers.

Earlier observations by various authors include dental erosions, periodontitis, erythema, aphthous ulcers, glossitis, etc. The overall findings in our study suggest that dental erosion and erythema were the most prominent oral manifestations followed by periodontitis, gingivitis, gingival ulcers, and glossitis.

In the present study, $88 \%$ of the patients with GI disturbance had one or other form of oral manifestations. So, on most occasions, the oral findings may serve as a clue or act as a valuable guide for the diagnosis of GI disorders.

\section{CONCLUSION}

In our study of 250 patients, oral manifestations were observed in $88 \%$ of the patients. Both soft tissue and hard tissue changes were evident. There was a high correlation between various GI disease and dental erosion, erythema at various sites of the oral cavity, oral ulcers, gingivitis, periodontitis, and glossitis.

Careful evaluation of oral cavity may unveil many GI disorders and help the patient by providing early diagnosis, which further facilitates the prognosis.

\section{REFERENCES}

1. Rosengard, Heather C. Oral manifestations of systemic diseases [Internet]. Medscape reference [cited 2016 July 27]. Available from: http://emedicine.medscape.com/ article/1081029overview\#a2

2. Daley TD, Armstrong JE. Oral manifestations of gastrointestinal diseases. Can J Gastroenterol 2007;21(4):241-244.

3. Vakil, N, van Zanten SV, Kahrilas P, Dent J, Jones R; Global Consensus Group. The Montreal definition and classification of gastroesophageal reflux disease: a global evidence-based consensus. Am J Gastroenterol 2006 Aug;101(8):1900-1920.

4. Patrick L. Gastroesophageal reflux disease (GERD): a review of conventional and alternative treatments. Altern Med Rev 2011 Jun;16(2):116-133. 
5. Romano C, Cardile S. Gastroesophageal reflux disease and oral manifestations. Ital J Pediatr 2014 Aug;40(Suppl 1): A73.

6. Ardizzone S. Ulcerative colitis. Orphanet encyclopedia; September 2003.

7. Elahi M, Telkabadi M, Samadi V, Vakili H. Association of oral manifestations with ulcerative colitis. Gastroenterol Hepatol Bed Bench 2012 Summer;5(3):155-160.

8. Dye BA, Kruszon Moran D, McQuilian G. The relationship between periodontal disease attributes and helicobacter pylori, Infection among adults in the United States. Am J Public Health 2002 Nov;92(11):1809-1815.

9. Al Asqah M, Al Hamoudi N, Anil S, Aljebreen AR, Al Haumoudi WK. Is the presence of helicobacter pylori in the dental plaque of patients with chronic periodontitis a risk factor for gastric infection? Can J Gastroenterol 2009 Mar;23(3):177-179.

10. Schroeder PL, Filler SJ, Ramirez B, Lazarchik DA, Vaezi MF, Richter JE. Dental erosion and acid reflux disease. Ann Intern Med 2009 Jun;122(1):809-815.

11. Barron RP, Carmichael RP, Marcon MA, Sàndor GK. Dental erosion in gastroesophageal reflux disease. J Can Dent Assoc 2003 Feb;69(2):84-89.

12. Fan-Hsu J. Evidence linking gastroesophageal reflux disease and dental erosion is not strong. J Am Dent Assoc 2009 Nov;140(11):1401-1402.
13. Huber MA. Gastrointestinal illnesses and their effects on the oral cavity. Oral Maxillofac Surg Clin North Am 2008 Nov;20(4):625-634.

14. Schroeder PL. Dental erosion and acid reflux disease. Ann Intern Med 1995 Jun;122(11):809-815.

15. Trost LB, McDonnell JK. Important cutaneous manifestations of inflammatory bowel disease. Postgrad Med J 2005 Sep;81(959):580-585.

16. Galbraith SS, Drowlet BA, Kogathasin S, Paller AS, Esterly NB. Asymptomatic inflammatory bowel disease presenting with mucocutaneous findings. Paediatrics 2005 Sep;116(3): e439-e444.

17. Van Dyke TE, Dowell R Jr, Offenbacher S, Snyder W, Hersh T. Potential role of microorganisms isolated from periodontal lesions in the pathogenesis of inflammatory bowel disease. Infect Immun 1986 Sep;53(3):671-677.

18. Katz J, Shenkman A, Stavropoulos F, Melzer E. Oral signs and symptoms in relation to disease activity and site of involvement in patients with inflammatory bowel disease. Oral Dis 2003 Jan;9(1):34-40.

19. Jose FA, Heyman MB. Extraintestinal manifestations of inflammatory bowel disease. J Pediatr Gastroentrol Nutr 2008 Feb;46(2):124-133.

20. Franch AM, Soriano YJ, Perez MGS. Dental management of patients with inflammatory bowel disease. J Clin Exp Dent 2010;2(4):191-195. 